Santé mentale au Québec

Prévalence et cooccurrence de cinq formes de maltraitance en lien avec la dépression et le trouble de la conduite chez les mères adolescentes

Prevalence and co-occurrence of five types of ill-treatment in relation with depression and conduct disorder in adolescent mothers Prevalencia y co-ocurrencia de cinco formas de maltrato relacionadas con la depresión y el trastorno de la conducta en las madres adolescentes

Prevalência e concomitância de cinco formas de maus-tratos em relação com a depressão e o transtorno da conduta nas mães adolescentes

Daniel Paquette, Mark Zoccolillo et Marc Bigras

Volume 32, numéro 2, automne 2007

Mosaïques de recherche (1) et Windigo II (2)

URI : https://id.erudit.org/iderudit/017796ar

DOI : https://doi.org/10.7202/017796ar

\section{Aller au sommaire du numéro}

\section{Éditeur(s)}

Revue Santé mentale au Québec

\section{ISSN}

0383-6320 (imprimé)

1708-3923 (numérique)

\section{Découvrir la revue}

\section{Citer cet article}

Paquette, D., Zoccolillo, M. \& Bigras, M. (2007). Prévalence et cooccurrence de cinq formes de maltraitance en lien avec la dépression et le trouble de la conduite chez les mères adolescentes. Santé mentale au Québec, 32(2), 37-58. https://doi.org/10.7202/017796ar
Résumé de l'article

Dans cet article, les auteurs s'interrogent sur la cooccurrence des différentes formes de maltraitance subie par les mères adolescentes durant leur enfance, dans le but de mieux comprendre la transmission intergénérationnelle de la maltraitance. Ils soulignent que les études sur la maltraitance subie par des adultes ou des adolescents durant leur enfance ne tiennent généralement pas compte de la cooccurrence des différentes formes de maltraitance. Les auteurs vérifient dans un premier temps, si les mères adolescentes se distinguent des femmes de la population générale pour la prévalence et la cooccurrence des différentes formes de maltraitance subie durant leur propre développement. Ils établissent ensuite la prévalence et la cooccurrence des formes de maltraitance en fonction des diagnostics de dépression majeure et du trouble de la conduite chez les mères adolescentes. 


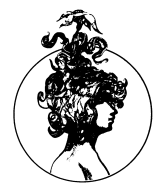

\title{
Prévalence et cooccurrence de cinq formes de maltraitance en lien avec la dépression et le trouble de la conduite chez les mères adolescentes
}

\author{
Daniel Paquette* \\ Mark Zoccolillo** \\ Marc Bigras ${ }^{\star \star *}$
}

\begin{abstract}
Dans cet article, les auteurs s'interrogent sur la cooccurrence des différentes formes de maltraitance subie par les mères adolescentes durant leur enfance, dans le but de mieux comprendre la transmission intergénérationnelle de la maltraitance. Ils soulignent que les études sur la maltraitance subie par des adultes ou des adolescents durant leur enfance ne tiennent généralement pas compte de la cooccurrence des différentes formes de maltraitance. Les auteurs vérifient dans un premier temps, si les mères adolescentes se distinguent des femmes de la population générale pour la prévalence et la cooccurrence des différentes formes de maltraitance subie durant leur propre développement. Ils établissent ensuite la prévalence et la cooccurrence des formes de maltraitance en fonction des diagnostics de dépression majeure et du trouble de la conduite chez les mères adolescentes.
\end{abstract}

L es études spécifiques sur la prévalence des différentes formes de maltraitance subies durant l'enfance et l'adolescence, et rapportées par les adolescents et les adultes de la population générale, demeurent rares. La grande majorité de la recherche a plutôt porté sur des populations cliniques ou encore d'étudiants universitaires (Brown et al., 1998). Aussi, les études sur les causes ou les conséquences de la maltraitance ne tiennent généralement pas compte de la cooccurrence des différentes formes de maltraitance, c'est-à-dire de la présence de plus d'une forme de mauvais traitement dans un même épisode de maltraitance ou de la succession de différentes formes de mauvais traitement lors d'épisodes distincts à propos du même enfant (Larrivée, 2005). Pourtant, les adultes, victimes durant leur enfance de formes multiples de mauvais

\footnotetext{
* Institut de Recherche pour le Développement Social des jeunes, Centre jeunesse de Montréal- Institut Universitaire.

** Hôpital de Montréal pour enfants.

*** Université du Québec à Montréal.
} 
traitement, rapportent significativement plus de problèmes d'ajustement à l'âge adulte que les victimes d'une seule forme de maltraitance (Higgins et McCrabe, 2001). De plus, certains chercheurs soutiennent que le phénomène des mauvais traitements multiples serait répandu. Dans une étude auprès d'un échantillon de convenance de la population générale, Paquette et al. (2004) ont démontré que parmi les femmes qui ont vécu de la maltraitance durant leur jeunesse (soit 110 sur 280), $50 \%$ des 110 avaient vécu au moins deux formes de maltraitance. Selon cette même étude, l'abus physique, l'abus émotionnel et la négligence physique sont plus souvent associés aux autres formes de maltraitance contrairement à la négligence émotionnelle et à l'abus sexuel.

La présente étude descriptive vérifie dans un premier temps, si les mères adolescentes se distinguent des femmes de la population générale quant à la prévalence et à la cooccurrence des différentes formes de maltraitance subies durant leur développement. Comparativement aux enfants des mères adultes, les enfants de mères adolescentes auraient un risque plus élevé (1) de mortalité/morbidité périnatale, (2) de problèmes cognitifs (par exemple, faible QI), (3) de problèmes socio-affectifs (par exemple, agressivité), (4) d'abus ou de négligence, (5) d'avoir des échecs scolaires, et (6) de devenir eux-mêmes des parents adolescents (Shapiro et Mangelsdorf, 1994). Les recherches montrent que les mères adolescentes seraient moins sensibles aux signaux de leur enfant que les mères adultes (Culp et al., 1988; Roosa et al., 1982). L'insensibilité maternelle vis-à-vis du nourrisson revêt une grande importance puisqu'elle est associée à un attachement insécurisant des enfants âgés de 12 à 18 mois (van den Boom, 1994; de Wolff et van IJzendoorn, 1997), et au développement de comportements extériorisés chez les enfants âgés entre 24 et 42 mois (Shaw et al., 1998). Les enfants des mères adolescentes auraient aussi un attachement insécurisant dans une proportion supérieure à un échantillon normatif de la population (Egeland et Sroufe, 1981 ; Lamb et al., 1987; Ward et Carlson, 1995).

Les études suggèrent généralement que les mères adolescentes seraient plus passives que les mères adultes (Elster et al., 1983; Hofferth, 1987). Elles seraient moins expressives, parleraient moins à l'enfant, feraient moins de comportements parentaux positifs et répondraient moins aux signaux des enfants que les mères adultes (Comfort et al., 1987; Culp et al., 1991 ; Garcia Coll et al., 1987; Hann et al., 1994; Jones et al., 1980; Kotagal, 1993; Landy et al., 1983; Roosa et al., 1982; Stevenson Barratt et Roach, 1995). Certains auteurs ont montré que les mères adolescentes sont plus négatives, restrictives et punitives que les mères adultes (Field et al., 1980; Hann et al., 1994; 
McAnarney et al., 1986). Certains chercheurs expliquent ces différences du comportement parental entre les mères adolescentes et les mères adultes non pas par l'âge maternel mais par la convergence d'autres facteurs tels le revenu et l'éducation (Buchholz et Korn-Bursztyn, 1993). De fait, les mères adolescentes sont généralement moins scolarisées et plus souvent monoparentales, pauvres et dépendantes de l'aide sociale que les mères adultes (Herrenkohl et al., 1998; Zuckerman et al., 1984). Elles ont aussi un réseau social plus limité (Buchholz et KornBursztyn, 1993). Or, certaines études contrôlées statistiquement pour le statut socioéconomique, ont montré qu'il n'y avait pas de différences sur le plan parental entre les mères adolescentes et les mères adultes (Garcia Coll et al., 1987; Sommer et al., 1993). Par contre, d'autres études utilisant des groupes comparables sur le plan socioéconomique ont rapporté des différences entre les deux groupes de mères, mais moindres que ce qui était attendu (Benasich et Brooks-Gunn, 1996; Chase-Lansdale et al., 1994; Wakschlag et al., 1996). Enfin, des études, qui ont fait un appariement selon la scolarité, le statut marital et le statut socioéconomique, montrent que le comportement parental des mères adolescentes se distingue nettement de celui des mères adultes (Elster et al., 1983; Garcia Coll et al., 1987). Dans une étude effectuée en 2001, Paquette et al. ont montré que les mères adolescentes sont moins passives et plus surcontrôlantes avec leur enfant âgé de 4-5 mois que les mères adultes de scolarité comparable. Démontrant que la sensibilité parentale n'est pas reliée à l'âge maternel, Paquette et al. (2001) ont proposé que les différences de sensibilité parentale entre les mères adolescentes et les mères adultes sont dues à des aspects particuliers de l'immaturité socio-affective. De fait, les mères adolescentes se distinguent des mères adultes par un vécu plus fréquent de maltraitance durant leur développement psychologique (Smith, 1996), de dépression (Osofsky et al., 1988), et de troubles de la conduite (Zoccolillo et al., 1997). Ces caractéristiques expliqueraient-elles les difficultés qu'ont les mères adolescentes à interpréter les émotions de l'enfant (Shapiro et Mangelsdorf, 1994), à se mettre à la place des autres et à être empathiques vis-à-vis leur enfant (Baranowski et al., 1990), à prédire les conséquences probables de leurs actions et à réguler ou contrôler leurs émotions augmentant ainsi le risque de maltraitance à l'égard de leur enfant (Gilligan et Belenky, 1980; Trad, 1995)?

\section{Prévalence et cooccurrence}

Nous examinons ici la prévalence et la cooccurrence des différentes formes de maltraitance en fonction des diagnostics de dépression majeure et du trouble de la conduite chez les mères adolescentes. Rauh 
et al. (1990) soutiennent que la dépression est le trait de personnalité qui semble avoir le plus grand impact sur les attitudes et les pratiques parentales. Les mères dépressives tendent à interpréter le comportement de l'enfant comme étant problématique (Richard et al., 1981). Elles ont tendance à interagir avec leurs enfants de manière désengagée ou intrusive, c'est-à-dire à être sous ou sur-stimulante (Cummings et Davies, 1994; Malphurs et al., 1996). De fait, elles ont relativement peu confiance en leurs habiletés parentales (Cummings et Davies, 1994) et sont plus enclines à utiliser des stratégies coercitives pour contrôler les enfants (Fendrich et al., 1990).

Le trouble de la conduite et les troubles émotionnels (dépression et anxiété) sont plus souvent présents ensemble que ce qui est attendu par le hasard (Zoccolillo, 1992). Les adolescents avec un trouble de la conduite diffèrent de leurs pairs par un certain nombre de caractéristiques. Ils ont généralement plus souvent une histoire familiale de psychopathologie et de maltraitance. Plusieurs études ont trouvé des taux relativement élevés de troubles de la conduite chez les mères adolescentes (Cassidy et al., 1996; Zoccolillo et al., 1997). D'autres ont montré que le trouble de la conduite est un prédicteur de la grossesse adolescente (Elster et al., 1990; Kessler et al., 1997; Kovacs et al., 1991). Selon Paquette et al. (2006), c'est la dépression maternelle et non le trouble de la conduite chez la mère adolescente qui est reliée au développement des comportements antisociaux (agression physique et extériorisation) chez les filles de trois ans. Ces auteurs soutiennent que l'agression maternelle constitue une variable intermédiaire entre la dépression maternelle et les comportements antisociaux des filles.

La recherche a montré que la dépression chez l'adulte est fortement associée à une histoire d'abus sexuel, et que les femmes sont plus souvent victimes de dépression et d'abus sexuel que les hommes (Whiffen et al., 2000). D'autre part, les études ont mis en évidence une relation entre l'abus sexuel et la grossesse adolescente $(\mathrm{Oz}$ et Fine, 1988 ; Smith, 1996; Roosa et al., 1997). Bien qu'un certain nombre de mères adolescentes aient été exposées à plusieurs formes de maltraitance (Smith, 1996), leurs effets sur leur santé mentale et leurs comportements parentaux sont encore mal connus, puisque les formes d'abus et de négligence sont rarement différenciées dans la recherche sur la transmission intergénérationnelle de la maltraitance (Spieker et al., 1996). 


\section{Méthodologie}

\section{Participantes; Échantillons de mères adolescentes}

Deux-cent quatorze (214) adolescentes enceintes de leur premier enfant ont été recrutées entre 1995 et 1998 afin de participer à une étude longitudinale du développement socio-affectif des enfants (projet $\mathrm{La}$ Mère Veille: Paquette et Morrisson, 1998). Le taux de refus s'est avéré inférieur à $10 \%$ chez les mères adolescentes. L'échantillon total se caractérise par un âge moyen à la naissance du premier enfant de 16,95 ans (E.T. $=1,09$; variant entre 14 et 19$)$ et une scolarité moyenne de 8,92 ans $($ E.T. $=1,42)$. Soixante et onze pour cent $(71 \%)$ sont des étudiantes. La principale source de leurs revenus est la suivante : 4,9\% travaillent ; 27,9\% reçoivent des prestations d'aide sociale; $52,5 \%$ sont aidées financièrement par leurs parents ; 3,4 \% par leur conjoint ; 3,9\% ont dit n'avoir aucun revenu; et 7,4\% autres revenus. Quarante-quatre pour cent $(43,9 \%)$ ont une histoire de trouble de la conduite et $37,8 \%$ une histoire de dépression majeure. Finalement, $30 \%$ sont d'une origine culturelle autre que nord-américaine.

Cent soixante (160) d'entre elles ont été recrutées à l'école Rosalie-Jetté lors d'une séance d'information en vue d'une inscription possible. Cette école de la Commission scolaire de Montréal aide spécialement des jeunes mères à terminer leur secondaire. Les adolescentes de cette école proviennent de tous les secteurs de la région métropolitaine de Montréal. Près du quart de l'échantillon total provient de services cliniques: 42 adolescentes ont été recrutées au Centre jeunesse de Montréal dans les quatre foyers de groupe pour mères en difficulté d'adaptation, et 12 adolescentes ont été recrutées par la clinique obstétrique de l'Hôpital de Montréal pour enfants.

L'échantillon «école» et l'échantillon «clinique» ne sont pas significativement différents pour l'âge moyen de la mère à la naissance de son premier enfant, pour les nombres moyens de symptômes de dépression et de trouble de la conduite (voir Tableau 1), et pour la proportion de celles qui ont un diagnostic de trouble de la conduite ou de dépression majeure. Ils ne se distinguent pas non plus pour la proportion d'immigrantes $(26,8 \%$ pour clinique versus $18,2 \%$ pour école). Par contre, les mères adolescentes de l'échantillon clinique sont en moyenne moins scolarisées, ont un QI verbal moyen significativement moindre (PPVT-R, Dunn et al., 1993) et ont vécu plus de placements en famille d'accueil ou en centres de réadaptation (entre la naissance et l'âge de 15 ans) que les mères adolescentes de l'échantillon «école» (voir Tableau 1). De plus, les mères adolescentes de l'échantillon «école»sont, en plus grande proportion, des étudiantes 
après l'accouchement $(76,9 \%$ versus $52,8 \%)$ et aidées financièrement par leurs parents (56\% versus $34 \%$ ) que dans l'échantillon clinique. Finalement, les mères adolescentes d'origine haïtienne sont plus nombreuses dans le groupe clinique que dans le groupe «école» $(22,0 \%$ versus $5,7 \%)$.

Tableau 1

Comparaison des moyennes (écarts-types) des caractéristiques personnelles entre les deux échantillons de mères adolescentes

\begin{tabular}{|l|l|l|l|}
\hline & $\begin{array}{l}\text { Échantillon } \\
\text { «clinique } \\
\text { mères adolescentes } \\
\mathrm{n}=54\end{array}$ & $\begin{array}{l}\text { Échantillon «école } \\
\text { de mères } \\
\text { adolescentes } \\
\mathrm{n}=160\end{array}$ & $\mathrm{t}(\mathrm{dl})$ \\
\hline Âge de la mère & $16,98(1,05)$ & $16,94(1,10)$ & $0,21(208)$ \\
\hline Scolarité & $8,44(1,21)$ & $9,08(1,45)$ & $2,87^{* *}(210)$ \\
\hline QI verbal & $95,10(16,01)$ & $101,86(12,27)$ & $2,70^{* *}(67,77)$ \\
\hline $\begin{array}{l}\text { Nbre de symptômes } \\
\text { de dépression }\end{array}$ & $5,78(2,52)$ & $5,70(2,54)$ & $0,21(208)$ \\
\hline $\begin{array}{l}\text { Nbre de symptômes } \\
\text { de trouble de la } \\
\text { conduite }\end{array}$ & $4,73(3,15)$ & $3,88(2,67)$ & $1,86(195)$ \\
\hline Nbre de placements & $2,68(2,69)$ & $0,88(2,97)$ & $3,75^{* * *}(67,56)$ \\
\hline
\end{tabular}

$* * \mathrm{p}<0,01 * * * \mathrm{p}<0,001$

Les prévalences de maltraitance dans l'échantillon de mères adolescentes sont comparées à celles d'un échantillon de convenance incluant 280 femmes âgées entre 14 et 44 ans, qui ont été recrutées via 31 organismes, centres, comités et écoles sélectionnés au hasard à partir du bottin téléphonique de la ville de Montréal. Elles se répartissent dans les catégories d'âge 14-19, 20-34 et 35-44 ans dans les mêmes proportions que dans la population générale (pour plus de détails, voir Paquette et al., 2004). Le taux de refus de participation est de $62 \%$. Cet échantillon de 280 femmes est caractérisé par un âge moyen de 29,22 ans $($ E.T. $=8,52)$ et une scolarité moyenne de 13,70 ans $($ E.T. $=3,66$; variant entre 8 et 27 ans). Vingt-six pour cent $(26,0 \%)$ n'ont pas complété leur secondaire alors que 33,5\% l'ont complété, $19,3 \%$ ont fait des études collégiales et $21,4 \%$ ont fait des études universitaires. Les études constituent la principale occupation pour (63\%) des sujets de l'échantillon. Le revenu personnel annuel brut se répartit de la manière suivante : 41,4\% gagnent moins de $\$ 10,000,38,7 \%$ de $\$ 10,000$ à $\$ 24,999,17,2 \%$ de $\$ 25,000$ à $\$ 39,999$, et $2,8 \%$ gagnent $\$ 40,000$ et 
plus. Finalement, 12,6\% sont d'une origine culturelle autre que nordaméricaine et $44,5 \%$ ont au moins un enfant à charge. Par certaines de ses caractéristiques (faible revenu, scolarité élevée, $63 \%$ aux études), cet échantillon de convenance se rapproche des échantillons de population étudiante universitaire, alors que par d'autres (âge moyen élevé, $45 \%$ ont un enfant à charge), il s'apparente davantage à un échantillon de la population générale d'adultes.

\section{Instruments}

Les instruments ont été soumis aux participantes avec le soutien d'une assistante de recherche. Le rôle de cette dernière était de s'assurer que les participantes comprennent bien chacune des questions du questionnaire et de l'entrevue.

L'histoire de maltraitance durant l'enfance et l'adolescence a été évaluée avec le Childhood Trauma Questionnaire (CTQ), un questionnaire développé par Bernstein et al. (1994) comprenant 70 items avec une échelle de type Likert en cinq choix de réponse (de $1=$ «jamais vrai » à $5=$ «très souvent vrai »). Le CTQ a été élaboré à partir d'une revue détaillée de la documentation sur la maltraitance et à partir aussi de l'expérience d'une entrevue structurée, le Childhood Trauma Interview, développée par les mêmes auteurs. Les analyses de Bernstein et al. (1994) auprès d'un échantillon de 286 patients avec un problème de dépendance à l'alcool ou à la drogue ont permis de dégager quatre facteurs, soit l'abus physique et émotionnel, la négligence émotionnelle, la négligence physique et l'abus sexuel. Par la suite, Bernstein et al. (1997) ont validé le CTQ avec une population de 398 patients adolescents psychiatrisés âgés de 12 à 17 ans. Cette étude a permis de séparer l'abus émotionnel de l'abus physique, et de mettre en évidence la présence de cinq facteurs qui expliquent $55 \%$ de la variance totale. L'abus émotionnel est lié à des attaques verbales à propos de la valeur de l'enfant en tant que personne ou de son sentiment de bien-être et à tout comportement humiliant, abaissant ou menaçant dirigé vers l'enfant par une personne plus âgée. L'abus physique est lié à des attaques physiques dirigées vers l'enfant par une personne plus âgée, attaques qui impliquent des blessures ou un risque de blessure. L'abus sexuel se rapporte à des contacts ou à des comportements de nature sexuelle entre un enfant et une personne plus âgée; le fait de contraindre explicitement l'enfant est un élément fréquent mais non essentiel de ce type d'abus. La négligence émotionnelle réfère à des situations où les personnes qui prennent soin de l'enfant ne répondent pas adéquatement à ses besoins psychologiques et affectifs de base, notamment l'amour, l'encouragement, le sentiment 
d'appartenance et le support. La négligence physique réfère à des situations où les personnes qui prennent soin de l'enfant ne répondent pas adéquatement à ses besoins physiques, dont le fait de nourrir, d'abriter, de superviser et de veiller à la santé et à la sécurité de l'enfant. Toutes les adolescentes de l'échantillon ont répondu au CTQ lorsqu'elles étaient enceintes de leur premier enfant. Les recherches antérieures ont démontré la validité des instruments rétrospectifs pour rapporter l'histoire d'abus et de négligence (Bifulco et al., 1997; Spieker et al., 1996). Le CTQ a été traduit en français et validé par Paquette et al. (2004). La consistance interne des échelles, évaluée par les alphas de Cronbach, est excellente et varie entre 0,79 et 0,94 . La stabilité temporelle des échelles, évaluée auprès de 12 sujets avec la corrélation de Pearson entre deux passations du questionnaire à trois semaines d'intervalle, est aussi excellente et varie entre 0,76 et 0,96 . Pour établir la présence ou non d'une histoire de maltraitance, Paquette et al. (2004) ont fait appel à cinq juges experts en maltraitance qui ont jugé le risque pour l'enfant à chacun des items. Les points de coupure utilisés sont la moyenne des scores (entre 1 et 5) arrondie à l'unité la plus près, soit 46 pour la négligence émotionnelle (17 items), 11 pour l'abus sexuel (5 items), 25 pour l'abus physique (10 items), 41 pour l'abus émotionnel (12 items) et 26 pour la négligence physique (8 items).

Les diagnostics de dépression majeure et de trouble de la conduite ont été établis durant la grossesse à partir de la version francophone du DIS (Robins et al., 1981) qui utilise les critères du DSM-IIIR. Un diagnostic de dépression était assigné lorsque la participante avait au moins un épisode de dépression majeure avant l'âge de 15 ans, et un diagnostic de trouble de la conduite lorsque la participante avait au moins trois symptômes de trouble de la conduite avant 15 ans. Les symptômes de trouble de la conduite font partie de l'évaluation du trouble de la personnalité antisociale. Les mères adolescentes de l'école et des foyers de groupe ont utilisé la version informatisée, et les informations ont été recueillies verbalement par une assistante avec la version papier-crayon pour les 12 adolescentes recrutées à l'hôpital.

\section{Résultats}

\section{Prévalence d'histoire d'abus et de négligence chez les mères adolescentes}

Le tableau 2 présente la prévalence de cinq formes de maltraitance dans l'échantillon clinique composé de 54 mères adolescentes et est comparée à un groupe de 160 mères adolescentes recrutées à l'école Rosalie-Jetté. Enfin, ces deux échantillons de mères adoles- 
centes sont comparés à un échantillon de 280 femmes de la population générale.

À noter que nous n'avons pas trouvé de différence significative des prévalences des formes de maltraitance entre les mères adolescentes 1416 ans et celles de 17-19 ans. Ensuite, les analyses de chi carré ont montré que les mères adolescentes de l'échantillon clinique sont plus nombreuses à avoir vécu une histoire de négligence émotionnelle, une histoire d'abus physique, une histoire de négligence physique et une histoire d'abus sexuel que celles qui proviennent de l'école. Ces deux échantillons ne se distinguent pas significativement sur l'abus émotionnel.

Tableau 2

Prévalence $(\%)$ des formes de maltraitance dans deux échantillons de mères adolescentes (14-19 ans) comparativement à un échantillon de femmes (14-44 ans) de la population générale

\begin{tabular}{lcccc}
\hline & $\begin{array}{l}\text { Échantillon } \\
\text { «clinique } \\
\text { de mères } \\
\text { adolescentes } \\
(\mathrm{n}=54)\end{array}$ & $\begin{array}{l}\text { Échantillon } \\
\text { «école }) \\
\text { mères } \\
\text { adolescentes } \\
(\mathrm{n}=160)\end{array}$ & $\begin{array}{l}\chi^{2}(\mathrm{dl}=1) \\
\text { «clinique } \\
\text { vs } \\
\text { «école }\end{array}$ & $\begin{array}{l}\text { Échantillon } \\
\text { de femmes de } \\
\text { la population } \\
\text { générale } \\
(\mathrm{n}=280)\end{array}$ \\
\hline Négligence émotionnelle & 43,4 & 28,3 & $4,16^{*}$ & 29,2 \\
Abus sexuel & 45,3 & 18,2 & $15,51^{* * *}$ & 19,9 \\
Abus physique & 37,7 & 18,8 & $7,99 * *$ & 13,9 \\
Abus émotionnel & 20,8 & 15,6 & 0,75 & 11,8 \\
Négligence physique & 17,0 & 4,4 & $9,11^{* *}$ & 2,8 \\
\hline
\end{tabular}

$* \mathrm{p}<0,05 * * \mathrm{p}<0,01 * * * \mathrm{p}<0,001$

Cinquante pour cent $(50 \%)$ des mères adolescentes (soit $62,3 \%$ pour le groupe clinique et $45,6 \%$ pour le groupe de l'école) et $39,6 \%$ des femmes de la population générale ont une histoire de maltraitance quelle qu'elle soit. Le tableau 2 montre aussi que les prévalences des cinq formes de maltraitance sont similaires chez les mères adolescentes de l'école et chez les femmes de la population générale.

\section{Cooccurrence des formes de maltraitance chez les mères adolescentes de l'école}

Parmi les adolescentes de l'école qui ont subi au moins une forme de maltraitance durant leur enfance ou adolescence, la négligence émotionnelle se retrouve dans $61,3 \%$ des cas, l'abus sexuel dans $40,4 \%$ des cas, l'abus physique et l'abus émotionnel dans respectivement $40,4 \%$ et $33,5 \%$ des cas, et enfin, la négligence physique dans $7,0 \%$ 
des cas (Tableau 3). Notons que ces proportions sont similaires à celles chez les femmes de la population générale.

Tableau 3

Pourcentages de cas de maltraitance en fonction du nombre de formes de maltraitance impliqués simultanément, et ce pour chaque forme de maltraitance, chez les femmes de la population générale et chez les mères adolescentes de l'école

\begin{tabular}{llrrrrrr}
\hline & & \multicolumn{7}{c}{ Nombre de formes de maltraitance } \\
& & \multicolumn{1}{c}{2} & \multicolumn{1}{c}{3} & \multicolumn{1}{c}{4} & \multicolumn{1}{c}{5} & TOTAL \\
\hline Négligence & femmes & 30,9 & 10,9 & 14,4 & 13,6 & 1,8 & 71,6 \\
émotionnelle & ados & 27,8 & 8,4 & 15,3 & 7,0 & 2,8 & 61,3 \\
Abus sexuel & femmes & 14,5 & 10,9 & 9,0 & 13,6 & 1,8 & 49,8 \\
& ados & 19,4 & 2,8 & 9,8 & 5,6 & 2,8 & 40,4 \\
Abus physique & femmes & 3,6 & 6,3 & 9,9 & 13,6 & 1,8 & 35,2 \\
& ados & 9,7 & 5,6 & 16,7 & 5,6 & 2,8 & 40,4 \\
Abus émotionnel & femmes & 2,0 & 5,4 & 9,0 & 11,8 & 1,8 & 30,0 \\
Négligence physique & ados & 1,4 & 8,4 & 13,9 & 7,0 & 2,8 & 33,5 \\
& femmes & 0 & 0 & 3,6 & 1,8 & 1,8 & 7,2 \\
& ados & 0 & 0 & 2,8 & 1,4 & 2,8 & 7,0 \\
\hline
\end{tabular}

Le tableau 3 permet de constater la forte cooccurrence des différentes formes de maltraitance, et ce, tout aussi bien chez les mères adolescentes de l'école que chez les femmes de la population générale. Deux formes de maltraitance sont moins associées à d'autres formes de maltraitance, soit la négligence émotionnelle et l'abus sexuel. Parmi les femmes qui ont vécu de la négligence émotionnelle, $45 \%$ des mères adolescentes $(27,8 / 61,3$, Tableau 3$)$ et $43 \%$ des femmes en général $(30,9 / 71,6)$ n'ont subi que cette forme de maltraitance. Parmi les femmes qui ont vécu de l'abus sexuel, $48 \%(19,4 / 40,4)$ des mères adolescentes et $29 \%(14,5 / 49,8)$ des femmes en général n'ont subi aucune autre forme de maltraitance. Chacune des trois autres formes de maltraitance est généralement associée à au moins deux autres formes de maltraitance. À noter enfin que la négligence physique n'est jamais observée seule sans cooccurrence avec d'autres formes de maltraitance.

\section{Prévalence d'histoire d'abus et de négligence chez les mères adolescentes en fonction de leur santé mentale}

Le tableau 4 présente la prévalence des différentes formes de maltraitance en fonction de la santé mentale des mères adolescentes («école» et «clinique»). Les prévalences d'abus physique et d'abus émotionnel sont significativement supérieures chez les mères 
adolescentes qui ont un diagnostic de trouble de la conduite ou un double diagnostic positif, c'est-à-dire les cas où il $\mathrm{y}$ a au moins un diagnostic de trouble de la conduite.

Tableau 4

Prévalence $(\%)$ des formes de maltraitance chez les mères adolescentes («école» et «clinique») en fonction des diagnostics de dépression majeure et de trouble de la conduite

\begin{tabular}{lccccc}
\hline & $\begin{array}{l}\text { Diagnostics } \\
\text { négatifs } \\
(\mathrm{n}=80)\end{array}$ & $\begin{array}{l}\text { Trouble de } \\
\text { la conduite } \\
(\mathrm{n}=40)\end{array}$ & $\begin{array}{l}\text { Dépression } \\
\text { majeure } \\
(\mathrm{n}=28)\end{array}$ & $\begin{array}{l}\text { Double } \\
\text { diagnostic } \\
\text { positif } \\
(\mathrm{n}=46)\end{array}$ & $\chi^{2}(\mathrm{dl}=3)$ \\
\hline $\begin{array}{l}\text { Négligence } \\
\text { émotionnelle }\end{array}$ & 27,8 & 40,0 & 25,0 & 41,3 & 4,07 \\
$\begin{array}{l}\text { Abus sexuel } \\
\begin{array}{l}\text { Abus physique } \\
\text { Abus émotionnel }\end{array}\end{array}$ & 12,5 & 28,2 & 25,0 & 47,8 & $19,12^{* * * *}$ \\
$\begin{array}{l}\text { Négligence } \\
\text { physique }\end{array}$ & 7,8 & 35,0 & 10,7 & 39,1 & $15,87^{* *}$ \\
\hline
\end{tabular}

$* * \mathrm{p}<0,01 * * * \mathrm{p}<0,001$

D'autre part, la prévalence d'abus sexuel chez les mères adolescentes qui n'ont aucune de ces deux psychopathologies est inférieure aux prévalences chez les mères adolescentes qui ont l'un ou l'autre des deux diagnostics. De plus, il y a un effet d'interaction puisque la prévalence d'abus sexuel chez les mères adolescentes qui ont les deux diagnostics, est significativement supérieure à celle des mères adolescentes qui ont l'un ou l'autre des deux diagnostics.

Bien que les mères adolescentes avec un diagnostic de trouble de la conduite semblent avoir une plus forte prévalence de négligence émotionnelle que les autres (Tableau 4), la différence ne s'avère pas significative.

\section{Cooccurrence des formes de maltraitance chez les mères adolescentes en fonction de leur santé mentale}

Parmi les mères adolescentes qui ont une histoire de maltraitance, le pourcentage de sujets ayant vécu de la négligence émotionnelle est élevé, et varie peu en fonction de l'histoire de santé mentale $(60,7$ à $71,0 \%$ : voir colonne Total du Tableau 5). Par contre, le pourcentage de mères qui ont vécu l'une des trois formes d'abus est supérieur lorsqu'il y a une histoire de dépression ou de trouble de la conduite, compa- 
rativement à celles qui n'ont aucun de ces diagnostics (voir colonne Total du Tableau 5).

\section{Tableau 5}

Pourcentages de cas de maltraitance en fonction du nombre de formes de maltraitance impliquées simultanément, et ce pour chaque forme de maltraitance, chez les mères adolescentes (« école» et «clinique») qui ont au moins une histoire de dépression ou au moins une histoire de trouble de la conduite comparativement à celles qui n'ont aucun de ces diagnostics

\begin{tabular}{llcccccc}
\hline & Problèmes de & \multicolumn{7}{c}{ Nombre de } & formes de & maltraitance & \\
& santé mentale & 1 & 2 & 3 & \multicolumn{1}{c}{4} & 5 & TOTAL \\
\hline Négligence & non & 38,7 & 6,5 & 22,6 & 3,2 & 0 & 71,0 \\
émotionnelle & dépression & 15,9 & 6,9 & 18,1 & 13,7 & 6,8 & 61,4 \\
& Tr. conduite & 16,1 & 7,2 & 17,8 & 8,9 & 10,7 & 60,7 \\
Abus sexuel & non & 12,9 & 6,5 & 9,7 & 3,2 & 0 & 32,3 \\
& dépression & 27,3 & 2,3 & 15,8 & 13,7 & 6,8 & 65,9 \\
& Tr. conduite & 23,2 & 1,8 & 14,3 & 10,7 & 10,7 & 60,7 \\
Abus physique & non & 6,5 & 9,7 & 16,1 & 3,2 & 0 & 35,5 \\
& dépression & 4,5 & 4,6 & 18,1 & 11,4 & 6,8 & 45,4 \\
Abus émotionnel & Tr. conduite & 5,4 & 9,0 & 21,4 & 10,7 & 10,7 & 57,2 \\
& non & 0 & 9,7 & 9,7 & 0 & 0 & 19,4 \\
& dépression & 0 & 2,3 & 15,8 & 13,7 & 6,8 & 38,6 \\
Négligence & Tr. conduite & 0 & 5,4 & 16,1 & 10,7 & 10,7 & 42,9 \\
physique & non & 0 & 0 & 9,7 & 3,2 & 0 & 12,9 \\
& dépression & 0 & 0 & 0 & 6,9 & 6,8 & 13,7 \\
& Tr. conduite & 0 & 0 & 0 & 3,6 & 10,7 & 14,3 \\
\hline
\end{tabular}

Nous avons aussi examiné la cooccurrence des différentes formes de maltraitance chez les mères adolescentes en fonction de la présence ou non d'un problème de santé mentale (dépression, trouble de la conduite). La cooccurrence de 4-5 formes de maltraitance est supérieure chez les mères adolescentes qui ont au moins l'un des deux problèmes de santé mentale, si on la compare à celle des mères adolescentes qui n'ont aucun des deux problèmes de santé mentale (Tableau 5). De plus, parmi les mères adolescentes qui ont une histoire de négligence émotionnelle sans cooccurrence avec les autres formes de maltraitance, les mères qui n'ont pas d'histoire de santé mentale $(38,7 \%)$ sont plus nombreuses que celles qui ont une histoire de dépression $(15,9 \%)$ ou une histoire de trouble de la conduite $(16,1 \%)$. Finalement, parmi les mères adolescentes qui ont une histoire d'abus sexuel sans cooccurrence avec les autres formes de maltraitance, les mères qui au moins une histoire de dépression $(27,3 \%)$ et celles avec au moins une histoire de 
trouble de la conduite $(23,2 \%)$ sont plus nombreuses que les mères qui n'ont aucun de ces deux problèmes de santé mentale $(12,9 \%)$.

\section{Discussion}

Les résultats ont démontré que les prévalences et les cooccurrences des cinq formes de maltraitance étudiées sont similaires chez les mères adolescentes recrutées dans une école spéciale, chez les femmes de la population générale. Toutefois, cette similarité n'infirme pas les résultats des études antérieures qui démontrent que les mères adolescentes ont plus souvent une histoire de maltraitance que les mères adultes (Smith, 1996), l'échantillon «école» de mères adolescentes n'étant pas représentatif de la population des mères adolescentes. L'échantillon «école» est plutôt représentatif des adolescentes enceintes qui ont décidé de terminer leurs études secondaires, ce qui n'est généralement pas le cas chez la plupart des mères adolescentes de la région métropolitaine de Montréal. Il est ainsi impossible de généraliser les résultats.

Par contre, les prévalences des cinq formes de maltraitance chez les mères adolescentes qui ont un suivi clinique sont nettement supérieures à celles des femmes de la population générale. Le 45,3\% de mères adolescentes du groupe clinique qui ont une histoire d'abus sexuel se rapproche des prévalences de 50-60\% rapportées dans d'autres études (Rainey, 1995). Ces résultats suggèrent que l'âge n'est peut-être pas un facteur primordial pour expliquer les différences souvent observées entre les mères adolescentes et les mères adultes. En plus d'une moindre scolarisation et d'un QI moins élevé, le groupe clinique se distingue du groupe école par un nombre plus élevé de placements en famille d'accueil ou en centres de réadaptation au cours des quinze premières années de la vie. Les différences obtenues entre les deux groupes de mères adolescentes, suggèrent l'importance de considérer ces variables pour expliquer la transmission intergénérationnelle de la maltraitance dans des recherches futures.

Les résultats montrent que la prévalence de maltraitance chez les mères adolescentes est fortement associée à leur histoire de santé mentale. Pour comprendre la cooccurrence des formes de maltraitance, il est très important de tenir compte de la cooccurrence de différents problèmes de santé mentale, particulièrement le diagnostic de trouble de la conduite. La prévalence de la dépression est associée significativement à la prévalence d'une seule forme de maltraitance, l'abus sexuel, alors que le diagnostic de trouble de la conduite est associé aux trois formes d'abus, physique, émotionnel et sexuel. Les mères adolescentes qui ont une histoire de dépression ont davantage tendance à rapporter 
avoir été victimes d'abus sexuel que celles sans une telle histoire. Les mères adolescentes qui ont une histoire de trouble de la conduite ont plus tendance à avoir vécu une ou plusieurs des trois formes d'abus que celles qui n'ont aucun diagnostic de trouble de la conduite. Finalement, les mères adolescentes, qui ont à la fois une histoire de dépression et une histoire de trouble de la conduite, sont encore plus à risque d'avoir été victimes d'abus sexuel durant leur enfance ou leur adolescence. Ces résultats apparaissent très importants car dans les études sur la transmission intergénérationnelle de la maltraitance, l'abus sexuel a été étudié en relation avec la dépression sans tenir compte du diagnostic de trouble de la conduite. Pourtant, ce dernier est non seulement associé à l'abus sexuel, mais aussi à deux formes d'abus qui ne sont pas nécessairement associées à la dépression. Ces mesures étant rétrospectives, nous ne pouvons établir la relation de cause à effet, et affirmer d'emblée que la maltraitance est à l'origine des problèmes de santé mentale identifiés chez les mères adolescentes. Des recherches futures devront vérifier l'hypothèse que la transmission intergénérationnelle de l'abus est due à des problèmes de santé mentale qui ont un impact sur le comportement parental de ces mères adolescentes. La tendance des mères adolescentes à être plus surcontrôlantes avec leurs nourrissons que les mères adultes (Paquette et al., 2001) pourrait sans doute être reliée aux formes d'abus, particulièrement l'abus sexuel dont elles ont été victimes durant leur propre développement. L'abus physique et l'abus émotionnel jouent probablement un rôle important dans la transmission intergénérationnelle de la maltraitance. Toutefois, Spieker et al. (1996) estiment que la transmission intergénérationnelle de la maltraitance est possiblement plus forte chez les mères adolescentes qui ont vécu une histoire d'abus sexuel. Pour répondre à ces questions, les mères adolescentes devront être comparées à un échantillon de mères adultes appariées pour le statut socioéconomique. Dans la présente étude, nous avions choisi un échantillon de femmes de la population générale simplement comme point de référence pour juger de l'ampleur du phénomène chez les mères adolescentes. Bien que l'échantillon de femmes ne soit pas représentatif de la population, il nous a permis de situer l'ampleur du phénomène de la maltraitance chez les mères adolescentes, en attendant un portrait plus précis de la population générale. Il nous semble très important d'établir dans des recherches futures, les prévalences des différentes formes de maltraitance dans la population générale, et non seulement auprès des populations étudiantes ou cliniques. 


\section{Conclusion}

L'examen de la cooccurrence des formes de maltraitance en fonction de la santé mentale a permis de constater que les trois formes d'abus - physique, émotionnel et sexuel - sont plus fréquentes et cooccurrentes chez les mères adolescentes qui ont un problème de santé mentale (dépression ou trouble de la conduite) par rapport à celles qui n'ont aucun des deux problèmes. Les prévalences de négligence physique se sont avérées trop faibles pour faire ressortir des liens avec la santé mentale. Alors que la négligence physique n'est jamais en occurrence simple, la négligence émotionnelle est plus souvent en occurrence simple chez les mères adolescentes et chez les femmes de la population générale; sa prévalence est supérieure chez les mères adolescentes sans aucun des deux problèmes de santé mentale évalués ici comparativement aux mères adolescentes qui ont l'un ou l'autre des problèmes de santé.

Parmi les femmes qui ont subi de l'abus sexuel, les mères adolescentes de l'école sont plus nombreuses (48\%) que les femmes de la population générale (29\%) à avoir subi l'abus sexuel sans autre forme de maltraitance. De plus, l'abus sexuel est plus souvent en occurrence simple chez les mères adolescentes qui ont un problème de santé mentale comparativement à celles qui n'ont pas au moins l'un des deux diagnostics évalués. La négligence émotionnelle et l'abus sexuel étant plus souvent des problématiques uniques que les autres formes de maltraitance, il semble que des facteurs très différents puissent être impliqués dans l'occurrence simple comparativement à la cooccurrence. Ce constat nous invite à explorer d'autres facteurs afin de mieux comprendre l'étiologie propre à chacune de ces deux formes de maltraitance.

\section{Références}

Baranowski, M. D., Schilmoeller, G. L., Higgins, B. S., 1990, Parenting attitudes of adolescent and older mothers, Adolescence, 25, 782-790.

Benasich, A. A., Brooks-Gunn, J., 1996, Enhancing maternal knowledge and child-rearing concepts: Results from an early intervention program, Child Development, 67, 1186-1205.

Bernstein, D. P., Ahluvalia, T., Pogge, D., Handelsman, L., 1997, Validity of the Childhood Trauma Questionnaire in an adolescent psychiatric population, Journal of the American Academy of Child and Adolescent Psychiatry, 36, 3, 340-348.

Bernstein, D. P., Fink, L., Handelsman, L., Foote, J., Lovejoy, M., Wenzel, K., SAPAREto, E., RugGiero, J., 1994, Initial reliability and validity of a 
new retrospective measure of child abuse and neglect, American Journal of Psychiatry, 151, 8, 1132-1136.

Bifulco, A., Brown, G. W., Lillie, A., Jarvis, J., 1997, Memories of childhood neglect and abuse : corroboration in a series of sisters, Journal of Child Psychology and Psychiatry, 38, 365-374.

BOYER, D., FinE, D., 1992, Sexual abuse as a factor in adolescent pregnancy and child maltreatment, Family Planning Perspectives, 24, 4-11.

Brown, J., Cohen, P., Johnson, J.G., SAlzinger, S., 1998, A longitudinal analysis of risk factors for child maltreatment: findings of a 17-year prospective study of officially recorded ans self-reported child abuse and neglect, Child Abuse and Neglect, 22, 11, 1065-1078.

BuchHolz, E. S., Korn-BurszTYn, C., 1993, Children of adolescent mothers : Are they at risk for abuse?, Adolescence, 28, 361-382.

CDIS Group, 1991-92, Computerized French Version of DIS III-R, Ottawa, University of Ottawa et Ottawa Civic Hospital.

Chase-Lansdale, P.L., Brooks-Gunn, J., Zamsky, E. S., 1994, Young AfricanAmerican multigenerational families in poverty: Quality of mothering and grandmothering, Child Development, 65, 373-393.

Comfort, M., WulfF, L., Smeriglio, V., 1987, Adolescent parenthood: Implications for care of the mother and child, Maryland Medical Journal, $36,955-958$.

Culp, R. E., Appelbaum, M. I., Osofsky, D. D., Levy, J. A., 1988, Adolescent and older mothers: Comparison between prenatal maternal variables and newborn interaction measures, Infant Behavior and Development, 11, 353-362.

Culp, R. E., CulP, A. M., Osofsky, J. D., Osofsky, H. J., 1991, Adolescent and older mothers' interaction patterns with their six-month-old infants, Journal of Adolescence, 14, 195-200.

Cummings, E. M., Davies, P. T., 1994, Maternal depression and child development, Journal of Child Psychology and Psychiatry and Allied Disciplines, 35, 1, 73-112.

De WolfF, M. S., Van IJZendoorn, M. H., 1997, Sensitivity and Attachment: a Meta-Analysis on Parental Antecedents of Infant Attachment, Child Development, 68, 4, 571-591.

Dunn, L. M., Thériault-Whalen, C. W., Dunn, L. M., 1993, Échelle de vocabulaire en images Peabody, Toronto, Psycan. 
Egeland, B., Sroufe, L. A., 1981, Attachment and early maltreatment. Child Development, 52, 44-52.

Elster, A. B., Ketterlinus, R., Lamb, M. E., 1990, Association between parenthood and problem behavior in a national sample of adolescents, Pediatrics, 85, 1044-1050.

Elster, A. B., McAnarney, E. R., Lamb, M. E., 1983, Parental behavior of adolescent mothers, Pediatrics, 71, 494-503.

Fendrich, M., Warner, V., Weissman, M. M., 1990, Family risk factors, parental depression and psychopathology in offspring, Developmental Psychology, 26, 40-50.

Field, T. M., Widmayer, S. M., Stringer, S., Ignatoff, E., 1980, Teenage, lower-class, black mothers and their preterm infants: An intervention and developmental follow-up, Child Development, 51, 426-436.

Garcia Coll, C. T., Hoffman, J., OH, W., 1987, The social ecology and early parenting of Caucasian adolescent mothers, Child Development, 58, 955-963.

Gilligan, C., BelenKy, M. F., 1980, A naturalistic study of abortion decisions, New Directions for Child Development, 7, 69-90.

Hann, D. M., Osofsky, J. D., Barnard, K. E., Leonard, G., 1994, Dyadic affect regulation in three caregiving environments, American Journal of Orthopsychiatry, 64, 263-269.

Herrenkohl, E. C., Herrenkohl, R. C., Egolf, B. P., Russo, M. J., 1998, The relationship between early maltreatment and teenage parenthood, Journal of Adolescence, 21, 291-303.

Higgins, D. J., McCABE, M. P., 2001, Multiple forms of child abuse and neglect: Adult retrospective reports, Aggression and Violent Behavior, 6 , 547-578.

Hofferth, S. L., 1987, The children of teen childbearers, in Hofferth, S. L., Hayes, C. D., eds., Risking the Future: Adolescent Sexuality, Pregnancy, and Childbearing, National Academy Press, Washington, DC.

Jones, F. A., Green, V., Krauss, D. R., 1980, Maternal responsiveness of primiparous mothers during the postpartum period: Age differences, Pediatrics, 65, 579-583.

Kessler, R. C., Berglund, P. A., Foster, C. L., Saunders, W. B., Stang, P. E., WALTERS, E. E., 1997, Social consequences of psychiatric disorder, II : Teenage parenthood, American Journal of Psychiatry, 154, 1405-1411.

Kotagal, U.R., 1993, Newborn consequences of teenage pregnancies, Pediatric Annals, 22, 2, 127-132. 
Kovacs, M., Krol, R. S. M., Voti, L., 1994, Early onset of psychopathology and the risk for teenage pregnancy among clinically referred girls, Journal of the American Academy of Child and Adolescent Psychiatry, 33, 1, 106-113.

LAMB, M. E., HopPS, K., ElSTER, A. B., 1987, Strange situation behavior of infants with adolescent mothers, Infant Behavior and Development, 10, 39-48.

Landy, S., Montgomery, J. S., Schubert, J., Cleland, J. F., Clark, C., 1983, Mother-infant interaction of teenage mothers and the effect of experience in observational sessions on the development of their infants, Early Child Development and Care, 10, 165-186.

LARRIVÉE, M.-C., 2005, L'abus physique et sa cooccurrence avec d'autres formes de mauvais traitements. Ampleur du phénomène et contributions à une étiologie différentielle, thèse de doctorat inédite, Université du Québec à Montréal.

Malphurs, J. E., Field, T. M., Larraine, C., Pickens, J., Pelaez-Nogueras, M., YANDO, R., Bendell, D., 1996, Altering withdrawn and intrusive interaction behaviors of depressed mothers, Infant Mental Health Journal, 17, 2, 152-160.

McAnarney, E. R., Lawrence, R. A., Ricciuti, H. N., Polley, J., Szilagyi, M., 1986, Interactions of adolescent mothers and their 1-year-old children, Pediatrics, 78, 585-590.

Osofsky, J. D., Hann, D. M., Peebles, C., 1993, Adolescent parenthood : Risks and opportunities for mothers and infants, in Zeanah, C.H., ed., Handbook of Infant Mental Health, The Guilford Press, New York, 106-119.

Oz, S., FINE, M., 1988, A comparison of childhood backgrounds of teenage mothers and their non-mother peers: A new formulation, Journal of Adolescence, 11, 251-261.

Paquette, D., Bigras, M., Emery, J., Parent, S., Zoccolillo, M., 2006, Transmission intergénérationnelle des problèmes de comportement des mères adolescentes à leur enfant: différences liées au sexe, in Verlaan, P., Déry, M., éds., Les conduites antisociales chez les filles: comprendre pour mieux agir, Presses de l'Université du Québec, 205-232.

Paquette, D., Bigras, M., Zoccolillo, M., Tremblay, R.E., Labelle, M.-E., AzAR, R., 2001, Comparaison de la sensibilité parentale entre des mères adolescentes et des mères adultes peu scolarisées, Revue de psychoéducation et d'orientation, 30, 2, 283-298.

Paquette, D., Laporte, L., Bigras, M., Zoccolillo, M., 2004, Validation de la version francophone du CTQ et prévalence de l'histoire de maltraitance, Santé mentale au Québec, 29, 1, 201-220. 
Paquette, D., Morrisson, D., 1998, Un profil descriptif de 100 mères adolescentes: étude préliminaire dans le cadre du projet La Mère Veille, Rapport de recherche pour l'IRDS.

Rainey, D. Y., Stevens-Simon, C., Kaplan, D. W., 1995, Are adolescents who report prior sexual abuse at higher risk for pregnancy?, Child Abuse and Neglect, 19, 10, 1283-1288.

Rauh, V. A., Wasserman, G. A., Brunelli, S. A., 1990, Determinants of maternal child-rearing attitudes, Journal of American Academy of Child and Adolescent Psychiatry, 29, 375-381.

REIS, J., 1988, Child-rearing expectations and developmental knowledge according to maternal age and parity, Infant Mental Health Journal, 9, 287-304.

Richard, K. M., Forehand, R., Wells, K. C., Griest, D. L., McMahon, R. J., 1981, A comparison of mothers of clinic-referred deviant, clinic-referred nondeviant and nonclinic children, Behaviour Research and Therapy, 19, 201-205.

Robins, L. N., Helzer, J. E., Croughan, J., RatlifF, K. S., 1981, The NIMH Diagnostic Interview Schedule: its history, characteristics and validity, Archives of General Psychiatry, 38, 381-389.

Roosa, M. W., Fitzgerald, H. E., Carson, N. A., 1982, Teenage and older mothers and their infants: A descriptive comparison. Adolescence, 27, $65,1-17$.

Roosa, M. W., Tein, J.-Y., Reinholtz, C., Angelini, P. J., 1997, The relationship of childhood sexual abuse to teenage pregnancy, Journal of Marriage and the Family, 59, 119-130.

Serbin, L. A., Peters, P. L., McAffer, V. J., Schwartzmann A. E., 1991, Childhood agression and withdrawal as predictors of adolescent pregnancy, early parenthood, and environmental risk for the next generation, Canadian Journal of Behavioral Science, 23, 318-331.

Shapiro, J. R., Mangelsdorf, S. C., 1994, The determinants of parenting competence in adolescent mothers, Journal of Youth and Adolescence, 23, 6, 621-641.

Shaw, D. S., Winslow, E. B., Owens, E. B., Vondra, J. I., Cohn, J. F., Bell, R. Q., 1998, The development of early externalizing problems among children from low-income families: a transformational perspective, Journal of Abnormal Child Psychology, 26, 2, 95-107.

Sмith, C., 1996, The link between childhood maltreatment and teenage pregnancy, Social Work Research, 20, 3, 131-141. 
Sommer, K., Whitman, T. L., Borkowski, J. G., Schellenbach, C., Maxwell, S., KeOGH, D., 1993, Cognitive readiness and adolescent parenting, Developmental Psychology, 29, 389-398.

Spieker, S. J., Bensley, L., McMahon, R. J., Fung, H., Ossiander, E., 1996, Sexual abuse as a factor in child maltreatment by adolescent mothers of preschool aged children, Development and Psychopathology, 8, 497-509.

Stevenson Barratt, M., Roach, M. A., 1995, Early interactive processes : Parenting by adolescent and adult single mothers, Infant Behavior and Development, 18, 97-109.

TRAD, P. V., 1995, Mental health of adolescent mothers, Journal of the American Academy of Child and Adolescent Psychiatry, 34, 2, 130-142.

VAN DEN BOOM, D. C., 1994, The influence of temperament and mothering on attachment and exploration: an experimental manipulation of sensitive responsiveness among lower-class mothers with irritable infants, Child Development, 65, 5, 1457-1477.

Wakschlag, L. S., Chase-Lansdale, P. L., Brooks-Gunn, J., 1996, Not just "ghosts in the nursery»: Contemporaneous intergenerational relationships and parenting in young African-American families, Child Development, 67, 2131-2147.

WARD, M. J., CARlson, E. A., 1995, Associations among adult attachment representations, maternal sensitivity, and infant-mother attachment in a sample of adolescent mothers, Child Development, 66, 69-79.

Whiffen, V. E., Thompson, J. M., Aube, J. A., 2000, Mediators of the link between childhood sexual abuse and adult depressive symptoms, Journal of Interpersonal Violence, 15, 10, 1100-1119.

ZocCOlillo, M., 1992, Co-occurrence of conduct disorder and its adult outcomes with depressive and anxiety disorder: A review, Journal of the American Academy of Child and Adolescent Psychiatry, 31, 547-556.

Zoccolillo, M., Meyers, J., Assiter, S., 1997, Conduct disorder, substance dependence, and adolescent motherhood, American Journal of Orthopsychiatry, 67, 1, 152-157.

Zuckerman, B. S., Walker, D.K., Frank, D. A., Chase, C., Hamburg, B., 1984, Adolescent pregnancy: Biobehavioral determinants of outcome, Journal of Paediatrics, 105, 6, 857-863. 


\section{ABSTRACT \\ Prevalence and co-occurrence of five types of ill-treatment in relation with depression and conduct disorder in adolescent mothers}

In this article, the authors reflect on co-occurrence of different types of maltreatment that adolescent mothers have suffered during their childhood in order to better understand intergenerational transmission of maltreatment. They underline that studies on maltreatment that adults or adolescents have been subjected to during their childhood generally do not take into account co-occurrence of different types of maltreatment. The authors first attempt to verify if adolescent mothers are different from women of the general population in regards to prevalence and cooccurrence of various types of maltreatment suffered during their own development. They then try to establish prevalence and co-occurrence of types of maltreatment in relation to diagnoses of major depression or conduct disorder in adolescent mothers.

\section{RESUMEN}

\section{Prevalencia y co-ocurrencia de cinco formas de maltrato relacionadas con la depresión y el trastorno de la conducta en las madres adolescentes}

Con el propósito de comprender mejor la transmisión intergeneracional del maltrato, en este artículo los autores se interrogan acerca de la co-ocurrencia de las diferentes formas de maltrato sufridas por las madres adolescentes durante su infancia. Resaltan que los estudios sobre el maltrato sufrido por los adultos o adolescentes durante su infancia generalmente no tienen en cuenta la co-ocurrencia de las diferentes formas de maltrato. En una primera etapa, los autores verifican si las madres adolescentes se distinguen de las mujeres de la población general en la prevalencia y la co-ocurrencia de las diferentes formas de maltrato sufrido durante su propio desarrollo. Enseguida establecen la prevalencia y co-ocurrencia de las formas de maltrato en función de los diagnósticos de depresión mayor y el trastorno de la conducta en las madres adolescentes. 


\section{RESUMO}

Prevalência e concomitância de cinco formas de maus-tratos em relação com a depressão e o transtorno da conduta nas mães adolescentes

Neste artigo, os autores questionam a concomitância das diferentes formas de maus-tratos sofridos pelas mães adolescentes durante a infância, com o objetivo de melhor compreender a transmissão intergeracional dos maus-tratos. Eles ressaltam que os estudos sobre os maus-tratos recebidos pelos adultos ou pelos adolescentes durante a infância geralmente não levam em consideração a concomitância das diferentes formas de maus-tratos. Os autores verificam, em um primeiro momento, se as mães adolescentes se distinguem das mulheres da população em geral para a prevalência e a concomitância das diferentes formas de maus-tratos recebidos durante seu próprio desenvolvimento. Eles estabelecem, em seguida, a prevalência e a concomitância das formas de maus-tratos em função dos diagnósticos de depressão grave e do transtorno de conduta nas mães adolescentes. 